\title{
Synthesis and characterization of mesoporous carbon spheres
}

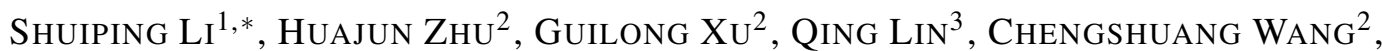 \\ QISHENG WU ${ }^{2}$, ZHANHONG WANG ${ }^{4}$ \\ ${ }^{1}$ College of Civil Science and Engineering, Yangzhou University, Yangzhou, 225127, P.R. China \\ ${ }^{2}$ School of Materials Science and Engineering, Yancheng Institute of Technology, Yancheng, 224051, P.R. China \\ ${ }^{3}$ School of Materials Engineering, Jinling Institute of Technology, Nanjing, 211169, P.R. China \\ ${ }^{4}$ Analysis and Testing Center, Yancheng Institute of Technology, Yancheng, 224051, P.R. China
}

\begin{abstract}
A three-step method was used for the synthesis of mesoporous carbon sphere (MCS) material: firstly, silica $\left(\mathrm{SiO}_{2}\right)$ nanoparticles were synthesized by the modified Stöber procedure; secondly, tetraethyl orthosilicate (TEOS) and cetyltrimethylammonium chloride (CTAC) were grafted onto $\mathrm{SiO}_{2}$ core nanoparticles to prepare $\mathrm{SiO}_{2} @ \mathrm{SiO}_{2}-\mathrm{CTAC}$ hard templates; lastly, MCS material was fabricated by carbonizing and selective leaching $\mathrm{SiO}_{2} /$ resorcinol-formaldehyde (RF) composites. The influence of the mass ratio of R-F/silica on the structure, morphology and crystal form was studied. The results indicate that the MCS materials have a uniform morphology. The increase of the mass ratio of R-F/silica can increase the specific surface area and pore volume. The three-step method provides a cost-effective procedure for the fabrication of MCS materials with uniform morphology.
\end{abstract}

Keywords: carbon materials; mesoporous materials; three-step method

\section{Introduction}

In recent years, there has been a considerable interest in the preparation of inorganic materials with spherical structure, which was due to their remarkable properties and chemical inertness [14]. Especially, mesoporous carbon sphere (MCS) materials with large surface area, appropriate pore volume and excellent mechanical stability, exhibit great potential to be used as a significant class of functional materials for catalysis, electrodes, sensors, etc. [5-8]. There have been several reports focused on the preparation of mesoporous carbon spheres. Li et al. [9] reported a novel approach to the synthesis of carbonaceous materials, which involved creation of mesoporoes in carbon precursor particles by imprinting with colloidal silica particles. Monodisperse resorcinol formaldehyde (RF) resin polymer spheres with finely tunable particle size ranging from $200 \mathrm{~nm}$ to $1000 \mathrm{~nm}$ were prepared by an extension of the Stöber method [10]. Choma et al. presented an extension of the Stöber method to synthesis of carbon particles, which had micropores, mesopores or both [11].

*E-mail: lishuiping2002@hotmail.com
In the current study, a novel process for synthesis of MCS materials with uniform morphology has been presented. Firstly, silica nanoparticles were synthesized by a modified Stöber procedure; secondly, hard templates of $\mathrm{SiO}_{2} @ \mathrm{~m}-\mathrm{SiO}_{2}-\mathrm{CTAC}$ were prepared through the cooperative formation mechanism of CATC and TEOS; finally, MCS materials were fabricated by stirring to generate $\mathrm{RF} /$ silica composites in one pot, after which carbonization and selective leaching were performed to obtain the MCS materials. To the best of our knowledge, no study has yet reported the synthesis of MCS materials through this three-step method. This procedure is cost-effective because the carbon source, surfactant and solvent are inexpensive. The obtained MCS materials presented an expected spherical structure and uniform size.

\section{Experimental}

\subsection{Materials}

Tetraethyl orthosilicate (TEOS) and resorcinol were purchased from Sinopharm Chemical Reagent Co., Ltd. (Shanghai, China). Hexadecyltrimethylammonium chloride (CTAC) 
was obtained from Sigma Aldrich Chemical Reagent Co., Ltd. (St. Louis, America). Aqueous ammonia, formaldehyde and ethanol were supplied by Yixing Tongsheng Chemical Reagent Co., Ltd. (Yixing, China). Hydrofluoric acid was purchased from Yellow River Fine Chemical Company (Xinxiang, China).

\subsection{Synthesis of $\mathrm{SiO}_{2}$ nanoparticles}

Silica nanoparticles were synthesized by a modified Stöber procedure using tetraethyl orthosilicate (TEOS) as silica source. $2.5 \mathrm{~mL}$ of aqueous ammonia was added to $25 \mathrm{~mL}$ of distilled water and stirred for 25 min under magnetic stirring at room temperature (Solution A). $1.5 \mathrm{~mL}$ of TEOS was dissolved in $25 \mathrm{~mL}$ of ethanol and also stirred for 25 min under magnetic stirring at room temperature (Solution B). Then, solution B was dropped into solution $\mathrm{A}$ and the system was kept stirring for $24 \mathrm{~h}$. The white precipitates $\left(\mathrm{SiO}_{2}\right)$ were separated by centrifugation, washed with ethanol and water, and dried at $105{ }^{\circ} \mathrm{C}$ for $24 \mathrm{~h}$.

\subsection{Preparation of $\mathrm{SiO}_{2} @ \mathrm{SiO}_{2}$-CTAC}

$0.17 \mathrm{~g}$ of CTAC was added to the solution containing distilled water $(30 \mathrm{~mL})$, aqueous ammonia $(0.45 \mathrm{~mL})$ and ethanol $(13 \mathrm{~mL})$ under stirring at room temperature. After $15 \mathrm{~min}, 0.1 \mathrm{~g}$ of $\mathrm{SiO}_{2}$ was added to the solution and the system was kept stirring for $60 \mathrm{~min}$. After that, $1.5 \mathrm{~mL}$ of TEOS was dropwise added to the solution under stirring at room temperature. The solution was kept under stirring for $6 \mathrm{~h}$. Then, the precipitates $\left(\mathrm{SiO}_{2} @ \mathrm{SiO}_{2}-\right.$ CTAC) were separated by centrifugation, washed with ethanol and water, and dried in an oven at $105{ }^{\circ} \mathrm{C}$ overnight.

\subsection{Fabrication of mesoporous carbon sphere materials}

$0.1 \mathrm{~g}$ of $\mathrm{SiO}_{2} @ \mathrm{SiO}_{2}-\mathrm{CTAC}$ was added to the solution containing distilled water $(200 \mathrm{~mL})$, aqueous ammonia $(1 \mathrm{~mL})$ and ethanol $(20 \mathrm{~mL})$ under stirring at room temperature. After $15 \mathrm{~min}$, $0.8 \mathrm{~g}$ of resorcinol was dropped into the solution and stirred for $30 \mathrm{~min}$. Then, $1 \mathrm{~mL}$ of formaldehyde was dropped into the solution and stirred for $15 \mathrm{~min}$. The solution was first stirred at $30{ }^{\circ} \mathrm{C}$ for $24 \mathrm{~h}$ and then at $100{ }^{\circ} \mathrm{C}$ for 24 . The precipitates $\left(\mathrm{SiO}_{2}\right.$ /resorcinol-formaldehyde - $\left.\mathrm{RF}\right)$ were separated by centrifugation, washed with ethanol and water, and dried under vacuum at $80{ }^{\circ} \mathrm{C}$ overnight.

$\mathrm{SiO}_{2} / \mathrm{RF}$ was heated to $600{ }^{\circ} \mathrm{C}$ at a heating rate of $2{ }^{\circ} \mathrm{C} \mathrm{min}{ }^{-1}$ and calcined for $6 \mathrm{~h}$ under argon atmosphere. After cooling to room temperature, $\mathrm{SiO}_{2}$ was removed through selective leaching method by hydrofluoric acid (HF, 4 wt.\%). The final product (MCS-10) was washed, centrifugalized and then dried at $80{ }^{\circ} \mathrm{C}$ for $24 \mathrm{~h}$ under vacuum. Other MCS materials (MCS-15 and MCS-20) were prepared at different molar ratios of R-F/silica, while the other process conditions were kept unchanged. The synthesis route of MCS materials is illustrated in Scheme 1.

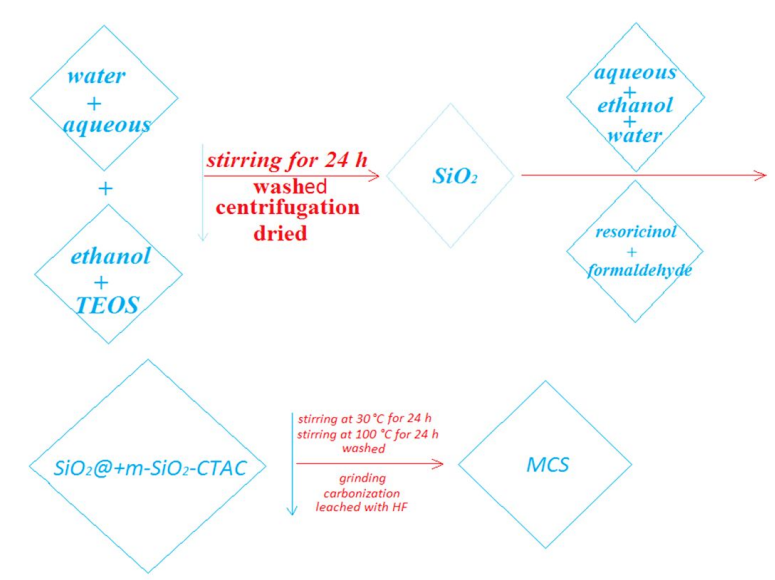

Scheme 1. Synthesis route of MCS materials.

\subsection{Characterization}

Attenuated total internal reflectance Fouriertransform infrared spectroscopy (ATR-IR) spectra were measured with a Tensor 37 instrument (Germany, Bruker). Each sample was scanned in the range of $400 \mathrm{~cm}^{-1}$ to $4000 \mathrm{~cm}^{-1}$ (ATR-test, 32 times scanning) with a resolution of $4 \mathrm{~cm}^{-1}$. Field emission scanning electron microscope (FE-SEM) images were recorded by a Hitachi SU 8000 microscope (Hitachi, Japan), and the fracture surfaces of the composites were sputter-coated with gold before observation. FE-SEM photo micrographs were obtained under conventional 
secondary electron imaging conditions at an accelerating voltage of $10 \mathrm{kV}$. High resolution transmission electron microscope (TEM) morphologies were observed by a JEM-2100F (JEOL, Japan) at an accelerating voltage of $200 \mathrm{kV}$. Samples of powders were suspended in ethanol using an ultrasonic horn, and small volumes of the suspension were deposited onto carbon-coated 400 mesh copper grids and dried under air. Small-angle X-ray scattering (SAXS) patterns were recorded on a X'Pert3 Powder (PANalytical, Netherlands) using $\mathrm{CuK} \alpha$ radiation $(\lambda=0.1540598 \mathrm{~nm}) . \mathrm{N}_{2}$ adsorptiondesorption isotherms at $77 \mathrm{~K}$ were measured with a Tristar II 3020 instrument (Micromeritics, America). The specific surface area was calculated by the Brunauer-Emmett-Teller (BET) method from the linear part of BET plot, according to IUPAC recommendations, using the adsorption isotherm (relative pressure $\mathrm{P} / \mathrm{P}_{0}=0.05$ to 0.20 ). The pore size distribution and pore volumes were calculated by the Barrett-Joyner-Halenda (BJH) method.

\section{Results and discussion}

FE-SEM images of MCS-10, MCS-15 and MCS-20 and EDS spectrum of MCS-20 material are shown in Fig. 1. As shown in the figure, all the MCS materials have a spherical morphology (Fig. 1a, Fig. 1b and Fig. 1c). MCS-10, MCS15 and MCS-20 materials exhibit particle sizes of $130 \mathrm{~nm}$ to $400 \mathrm{~nm}, 270 \mathrm{~nm}$ to $400 \mathrm{~nm}$ and $290 \mathrm{~nm}$ to $470 \mathrm{~nm}$, respectively. The SEM images of $\mathrm{SiO}_{2}$ nanoparticles synthesized using the modified Stöber method are displayed in Fig. 1a, which shows that the size of $\mathrm{SiO}_{2}$ particles ranges from $300 \mathrm{~nm}$ to $700 \mathrm{~nm}$. The sphere sizes of MCS-20 materials are more uniform and larger than those of MCS-10 and MCS-15, which indicates that a high $\mathrm{R}-\mathrm{F} / \mathrm{silica}$ ratio may result in the formation of $\mathrm{SiO}_{2}$ nanoparticles with uniform size. The EDS spectrum (Fig. 1d) shows that carbon is the main element in MCS-20. The peak of $\mathrm{Cu}$ element can be assigned to the copper grid. TEM morphologies of MCS-10, MCS-15 and MCS-20 and microstructure of mesoporous of MCS-20 materials are shown in Fig. 2. It can be seen that all samples exhibit a homogenous size distribution and similar dimensions (Fig. 2a, Fig. 2b and Fig. 2c). Moreover, all MCS materials exhibit an expected excellent spherical morphology. The sphere sizes of MCS materials are close to each other. MCS-10 materials have a particle size of $220 \mathrm{~nm}$ to $300 \mathrm{~nm}$. It should be noticed that the particle size of MCS10 observed by TEM is lower than the size of MCS-10 observed in the SEM images owing to the high resolution of TEM, which can clear distinguish the boundary of MCS particles. However, the TEM image of the microstructure of MCS-20 material presents a disordered mesoporous structure (Fig. 2d), which may result in a low specific surface area. These results indicate that the mass ratio of R-F/silica in the investigated range do not impart any obvious influence on the morphology of MCS materials.

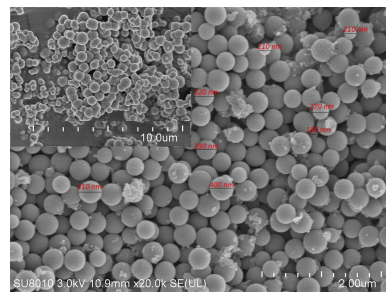

(a)

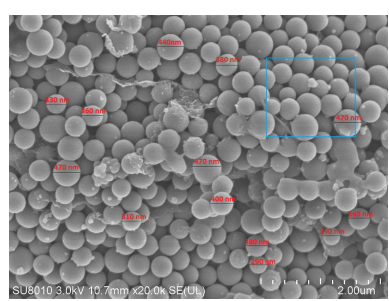

(c)

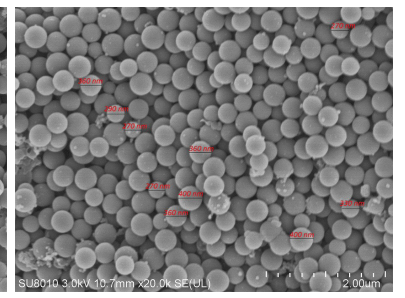

(b)

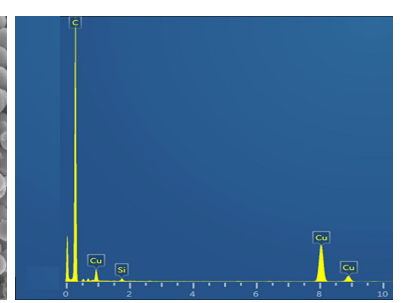

(d)
Fig. 1. FE-SEM images of MCS-10 (a), MCS-15 (b), and MCS-20 (c) materials, and EDS spectrum of MCS-20 material (d).

Fig. 3 shows the ATR-IR spectra of MCS-10, MCS-15 and MCS-20 materials. It can be seen that two weak characteristic peaks are present in all these three spectra. The peak at $1588 \mathrm{~cm}^{-1}$ can be assigned to the vibration of $\mathrm{C}=\mathrm{C}$, which is the characteristic peak of carbon materials. The appearance of the weak peak at $1084 \mathrm{~cm}^{-1}$, which is attributed to the stretching vibration of $\mathrm{Si}-\mathrm{O}-\mathrm{Si}$, indicates that there are few residues of $\mathrm{SiO}_{2}$ 
Table 1. Specific surface area, average pore size and pore volume of MCS materials.

\begin{tabular}{cccc}
\hline & $\begin{array}{c}\text { Specific surface area } \\
{\left[\mathrm{m}^{2} / \mathrm{g}\right]}\end{array}$ & $\begin{array}{c}\text { average pore size } \\
{[\mathrm{nm}]}\end{array}$ & $\begin{array}{c}\text { pore volume } \\
{\left[\mathrm{cm}^{3} / \mathrm{g}\right]}\end{array}$ \\
\hline \hline MCS-10 & 298 & 11.9 & 0.42 \\
MCS-15 & 435 & 10.1 & 0.46 \\
MCS-20 & 502 & 9.7 & 0.52 \\
\hline
\end{tabular}

in the MCS materials. This result is in accordance with the EDS spectrum, which is shown in Fig. 1d.

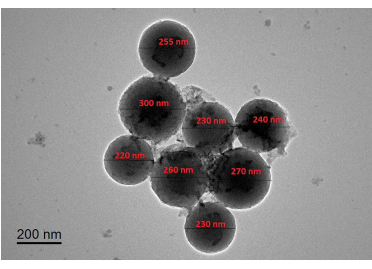

(a)

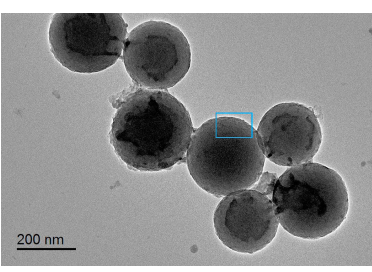

(c)

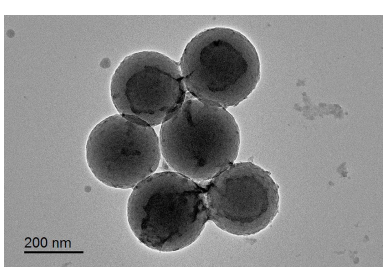

(b)

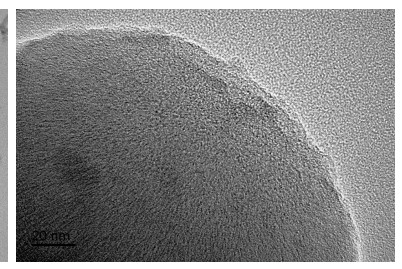

(d)
Fig. 2. TEM images of MCS-10 (a), MCS-15 (b), and MCS-20 (c) materials, and a pore structure of MCS-20 material.

$\mathrm{N}_{2}$ adsorption-desorption isotherms at $77 \mathrm{~K}$ of the MCS materials are shown in Fig. 4. The isotherms of MCS materials are all typical of IV isotherms with hysteresis loop, which results from capillary condensation in mesopores. These isotherms show a capillary condensation at a $\mathrm{P} / \mathrm{P}_{0}$ of 0.6 to 0.9 , which is characteristic of mesoporous materials [8]. Actually, type IV isotherms are typical isotherms of mesoporous materials according to the IUPAC convention [12]. However, the $\mathrm{N}_{2}$ adsorption-desorption isotherms do not show an obvious increase in adsorption at a relative pressure $\mathrm{P} / \mathrm{P}_{0}$ of 0.3 to 0.4 . This slow increase may exhibit a relative low pore volume [13]. The specific surface areas, average pore sizes and pore volumes of MCS materials are listed in Table 1. Table 1 shows that the specific surface areas of MCS-10,
MCS-15 and MCS-20 materials are $298 \mathrm{~m}^{2} / \mathrm{g}$, $435 \mathrm{~m}^{2} / \mathrm{g}$ and $502 \mathrm{~m}^{2} / \mathrm{g}$, respectively. The corresponding average pore size and pore volume are $11.9 \mathrm{~nm}, 10.1 \mathrm{~nm}$ and $9.7 \mathrm{~nm}$, and $0.42 \mathrm{~cm}^{3} / \mathrm{g}$, $0.46 \mathrm{~cm}^{3} / \mathrm{g}$ and $0.52 \mathrm{~cm}^{3} / \mathrm{g}$, respectively. These results reveal that increasing the mass ratio of $\mathrm{R}-\mathrm{F} /$ silica can improve the specific surface area and pore volumes but decrease the average pore sizes.

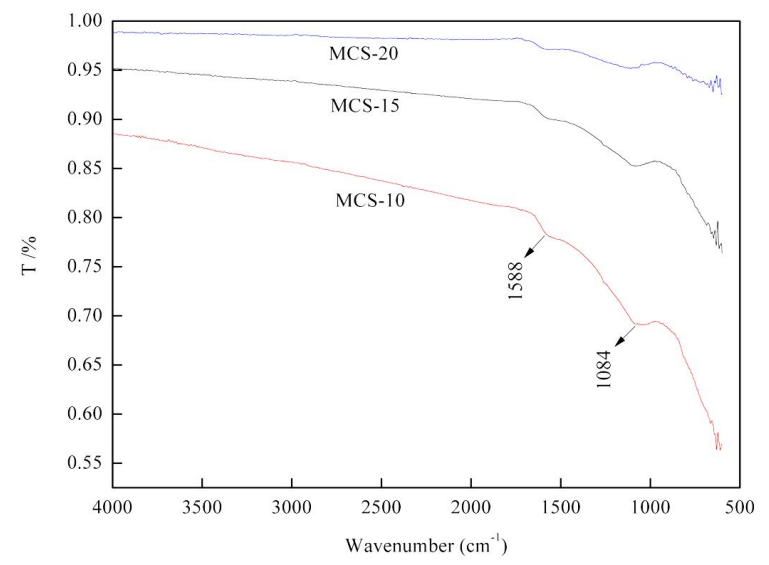

Fig. 3. ATR-IR spectra of MCS materials.

The mesostructural properties of MCS materials were also evaluated by SAXS (Fig. 5). The SAXS patterns of MCS materials present an apparent diffraction peak around $2 \theta=0.85^{\circ}$, indicating that all the samples have mesoporous pore walls [14]. The patterns do not show an obvious diffraction intensities decrease and diffraction peaks broadening owing to the disordered mesoscopic structure. This result demonstrates that the adjustment of mass ratio of R-F/silica exhibits no obvious influence on the crystal form of MCS materials. 


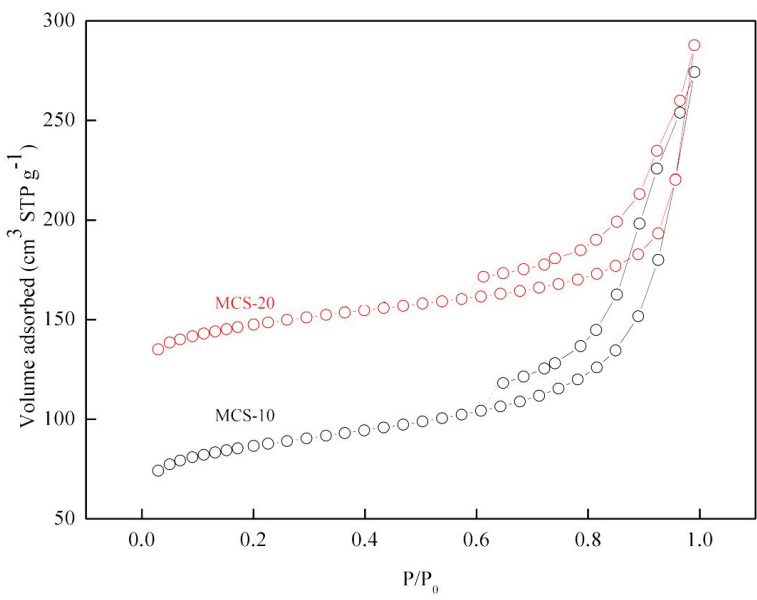

Fig. 4. Nitrogen sorption isotherms of MCS-10 and MCS-20 materials.

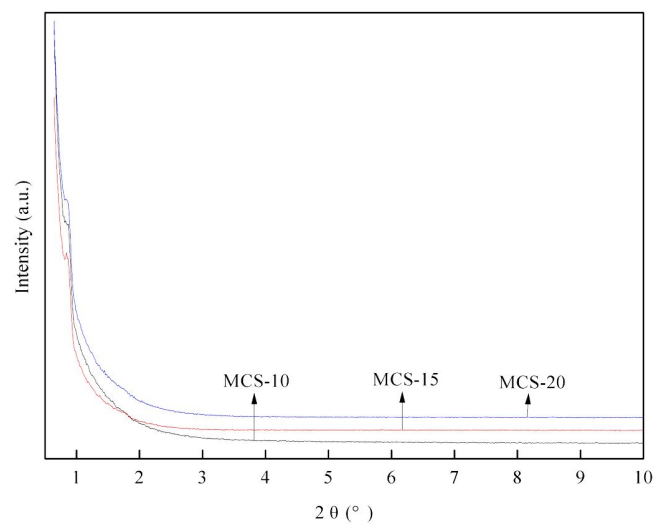

Fig. 5. SAXS patterns of MCS materials.

\section{Conclusions}

MCS materials were synthesized by threestep method: firstly, a modified Stöber method was used to prepare $\mathrm{SiO}_{2}$ nanoparticles; secondly, $\mathrm{SiO}_{2} @ \mathrm{SiO}_{2}-\mathrm{CTAC}$ hard templates were fabricated by grafting of TEOS and CTAC onto the surface of $\mathrm{SiO}_{2}$ nanoparticles; lastly, hard templates were coated with resorcinol and formaldehyde, followed by carbonization and selective leaching to remove $\mathrm{SiO}_{2}$ and prepare mesoporous carbon sphere (MCS) materials. The influence of the mass ratio of R-F/silica on the mesostructural properties of MCS materials was investigated using high resolution transmission electron microscope and $\mathrm{N}_{2}$ adsorption-desorption isotherms at $77 \mathrm{~K}$.
The results indicated that increasing the mass ratio of R-F/silica can improve the specific surface area and pore volume but decrease the average pore size. The mass ratio of R-F/silica had no obvious influence on the morphology and crystal form of the MCS materials. However, TEM images showed a disordered mesoporous structure, resulting in a low specific surface area.

\section{Acknowledgements}

The authors gratefully acknowledge the financial support of this study by the National Natural Science Foundation of China (51603179), the Six Talent Peaks Project in Jiangsu Province (2017-GDZB-053 and 2016-XCL-070), and the Science and Technology Project from the Ministry of Housing and Urbar-Rural Development of the People's Republic of China 2019-K-061.

\section{References}

[1] Zhou J., He J., Zhang C., Wang T., Sun D., Di Z., WANG D., Mater. Charact., 61 (2010) 61, 31.

[2] Wilgosz K., Chen X., KierzeK K., МAChNikOWski J., KALEnCZUK R.J., Mijowska E., Nanoscale Res. Lett., 7 (2012), 269.

[3] Yan Z., Xie J., Zong S., Zhang M., Sun Q., CHEN M., Electrochim. Acta, 109 (2013), 256.

[4] Zhang L., Kim J., Dy E., Ban S., Tsay K.C., KaWAi H., Shi Z., Zhang J., Electrochim. Acta, 108 (2013), 480.

[5] Guo L., Zhang L., Shi J., Mater. Lett., 65 (2011), 1.

[6] Tian Y., Zhong S., Zhu X., Huang A., Chen Y., WANG X., Mater. Lett., 161 (2015), 656.

[7] Wang D., Fu A., Li H., Wang Y., GuO P., LiU J., ZHAO X.S., J. Power Sources, 285 (2015), 469.

[8] Zhong S., Huang W., Tian Y., Wang X., Mater. Lett., 179 (2016), 86.

[9] Li Z., Jaroniec M., J. Am. Chem. Soc., 123 (2011), 9208.

[10] LiU J., Qiao S.Z., LiU H., Chen J., Orpe A., ZhaO D., LIU Q.G., Angew. Chem. Int. Edit., 50 (2011), 5947.

[11] Choma J., Jamiola D., AugustyneK K., MARSZEWSKI M., GAO M., JARONIEC M., J. Mater. Chem., 22 (2012), 12636.

[12] Rojas F., Kprnhauser I., Felipe C., Esparza J.M., Cordero S., Dominguez A., RicCArdo J.L., Phys. Chem. Chem. Phys., 4 (2002), 2346.

[13] Li S., Ww Q., Lu G., Zhang C., LiU X., Cui C., YAN Z., J. Mater. Eng. Perform., 22 (2013), 3762.

[14] LiU T., Lai D., Feng X., Zhu H., Chen J., Ceram. Int., 39 (2013), 3947. 